\title{
Recurrent Primary Peritoneal Low Grade Serous Adenocarcinoma
}

National Cancer Institute

\section{Source}

National Cancer Institute. Recurrent Primary Peritoneal Low Grade Serous

Adenocarcinoma. NCl Thesaurus. Code C156454.

The reemergence of primary peritoneal low grade serous adenocarcinoma after a period of remission. 